\title{
Iterative Development of an Innovative Smartphone- Based Dietary Assessment Tool: Traqq
}

\author{
Desiree A. Lucassen ${ }^{1}$, Elske M. Brouwer-Brolsma ${ }^{1}$, Anne M. van de Wiel ${ }^{1}$, Els Siebelink ${ }^{1}$, Edith J. M. Feskens ${ }^{1}$ \\ ${ }^{1}$ Division of Human Nutrition and Health, Wageningen University and Research
}

\section{Corresponding Author}

Desiree A. Lucassen

desiree.lucassen@wur.nl

\section{Date Published}

March 19, 2021

\section{Citation}

Lucassen, D.A., Brouwer-Brolsma, E.M., van de Wiel, A.M., Siebelink, E., Feskens, E.J.M. Iterative Development of an Innovative Smartphone-Based Dietary Assessment Tool: Traqq. J. Vis. Exp. (169), e62032, doi:10.3791/62032 (2021).

DOI

$10.3791 / 62032$
URL

jove.com/video/62032

\section{Abstract}

To collect dietary intake data in a fast and reliable manner, a flexible and innovative smartphone application (app) called Traqq was developed (iOS/Android). This app can be used as a food record and 24-h recall (or shorter recall periods). Different sampling schemes can be created on either prespecified or random days/times within a predetermined period for both methods, with push notifications to urge the participants to register their food intake. In case of non-response, notifications are automatically rescheduled to ensure complete data collection. For use as a food record, respondents can access the app and log their food intake throughout the day. Food records close automatically at the end of the day; recalls close after submission of the consumed items. The recall as well as the food record module provide access to an extensive food list based on the Dutch food composition database (FCDB), which can be accustomed to fit different research purposes. When selecting a food item, respondents are simultaneously prompted to insert portion size, i.e., in household measures (e.g., cups, spoons, glasses), standard portion sizes (e.g., small, medium, large), or weight in grams, and eating occasion/time of consumption. Portion size options can be adjusted, e.g., only entry in grams in case of a weighed food record or time of consumption instead of eating occasion). The app also includes a My Dishes function, which allows the respondent to create their own recipes or product combinations (e.g., a daily breakfast) and only report the total quantity consumed. Subsequently, the app accounts for yield and retention factors. The data are stored on a secure server. If desired, additional questions, i.e., in general or those related to specific food items or eating occasions can be incorporated. This paper describes the development of the system (app and backend), including expert evaluations and usability testing. 


\section{Introduction}

Accurate dietary assessment is crucial to ensure the quality of studies on the role of nutrition in health and disease prevention. Currently, such studies generally use established self-report dietary assessment methods, i.e., food frequency questionnaires, $24-\mathrm{h}$ recalls (24hRs), and/or food records ${ }^{1}$. Despite the fact that these methods are of major importance for nutrition research, they also possess various drawbacks, e.g., memory-related bias, social desirability bias, and are burdensome for the respondent as well as the researcher ${ }^{1,2}$. Recent technological inventions now offer the opportunity to overcome these drawbacks. During the past years, various research groups seized this opportunity and developed web-based and smartphone-based dietary assessment tools for nutrition research that address some of these known drawbacks (see Eldridge et al. ${ }^{3}$ for an extensive overview of web- and smartphone-based tools), i.e., reduce causes of error, improve user-friendliness, and decrease the participant's and researcher's burden ${ }^{1}$.

Nevertheless, the number of fully automated and validated smartphone applications (apps) that are appropriate for nutrition research is still limited. Most of the available dietary assessment apps (i.e., commercially or developed for research) are either not fully automated (i.e., require manual coding of food items) or are not (well) validated ${ }^{3}$. Moreover, most available validated apps have been developed for one specific research purpose and use in a specific country; due to rather fixed designs, re-using such apps for other research purposes or in other countries seems challenging $3,4,5,6,7,8$. Finally, despite the availability of food record-based apps, to date, no recall-based apps appear to exist yet. Although food records are prone to reactivity bias, i.e., respondents may alter their food intake due to the awareness that they are being observed ${ }^{2,9}$, this is not the case for recalls, which emphasizes the need for the development of a validated recall-based $\mathrm{app}^{10}$. An innovative dietary assessment app called Traqq was developed for use in the Netherlands which can be used as a food record as well as a recall, depending on the research question ${ }^{1}$.

Besides the possibility to alternate between the food record option and recall option, this app also differs from other dietary assessment tools because of its flexible nature. Specifically, regarding the food list, portion size estimates, sampling schemes, and the possibility to incorporate additional questions. The level of flexibility in the system enables tailoring to multiple research purposes that require accurate assessment of dietary behaviors. Currently, the app is in the process of being validated and will be ready to be used in various types of nutrition related research. The app can also be used, and perhaps further improved for use, in nutritional intervention programs to measure and influence dietary behaviors. As the development of reliable dietary assessment tools is challenging, and reports on these processes are scarce, especially with respect to user and expert involvement ${ }^{3,11,12}$, this paper provides a detailed overview on how different information sources were integrated in the systematic and iterative development of this smartphone-based dietary assessment app. The process incorporates theory, expert consultation, and user engagement.

\section{Protocol}

NOTE: All the procedures including human participants were conducted in a non-invasive manner by means of mostly qualitative research methods. Informed consent was obtained from all participants before the start of the evaluations. This protocol describes the iterative developmental process that 
can be roughly divided into four stages in which stages 1-3

are intertwined (Figure 1).

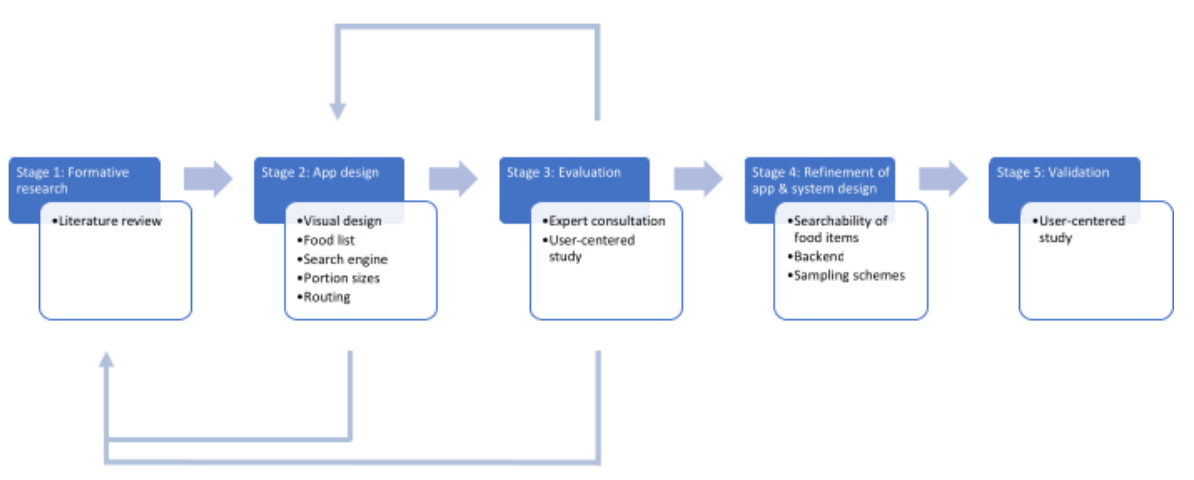

Figure 1: Overview of the stages of the iterative development process of the app. The development process consisted of five stages in total. However, the process was iterative which means that stages 1 through 3 were intertwined. Please click here to view a larger version of this figure.

\section{Conduct extensive formative research in preparation of the actual developmental process.}

1. Perform desktop research exploring existing weband smartphone-based dietary assessment tools, with special attention to features known to be of key importance for accurate food intake data collection, i.e., method of food entry (including the food list and underlying FCDB) and portion size estimations.

2. Inspect existing web- and smartphone-based dietary assessment tools focusing on aspects such as dietary assessment methodology, information provision, reliability, search engine, and implemented features (e.g., images, barcode scanner, recipe functions).

3. Consult experts in the field of dietary assessment.

NOTE: Results of the desktop research and inspection of existing tools were discussed with experts in the field of dietary assessment, leading to a draft design plan for the development of the app. This draft design plan was evaluated by the experts and further improved as required.

\section{Design the dietary assessment app}

1. Create the visual design of the app considering important aspects such as animation, branding, color, layout, and typography ${ }^{20}$.

NOTE: As space, color, fonts, graphics, and interface elements highlight content and convey interactivity, it is essential to incorporate elements facilitating the functionality of the app.

2. Select a trustworthy FCDB (here, NEVO) to facilitate nutrient calculations of the collected food intake data ${ }^{14}$.

3. Create a food list by critically evaluating the description of the food items mentioned in the FCDB. 
NOTE: FCDBs are mostly developed for professional use; food descriptions are often complex and hinder searchability (e.g., "margarine low-fat $35 \%$ fat $<10 \mathrm{~g}$ of saturated fats unsalted"23).

4. Formulate search engine requirements; consider the use of punctuation marks, foreign names, misspellings, different search terms, and ranking of search results to facilitate searchability of food items.

5. Select portion size estimation (aid) by evaluating various existing dietary assessment tools and field testing of suitable options.

6. Design routing within the app to ensure that the user's navigation through the app is logical, predictable, and easy to follow.

7. Design backend features and requirements to control the app; include functions related to overall project management, project-specific management (e.g., participants, invitations, data collection), and user management (e.g., authorizations).

\section{Evaluations by researchers}

NOTE: Following each upgrade, the app was tested by nutrition scientists and research dieticians with expertise in dietary assessment (in-house testing) to verify whether functionalities improved as anticipated. The following instructions are to be executed by researchers.

1. Conduct expert evaluation by means of cognitive walkthroughs to simulate a first-time user experience so that the experts can explore the app individually and without guidance ${ }^{28}$. Ensure that the cognitive walkthroughs consist of the following steps.
1. Make sure that the expert completes a general questionnaire inquiring about the brand and type of smartphone.

2. Install the app on the expert's smartphone.

NOTE: To ensure proper installation and functioning and minimize the risk of interruptions during the evaluation, it is recommended that the researcher first verifies the app's functionality.

3. Instruct the expert on test procedures in which each expert is asked to take on the role of first-time user (i.e., research participant). Emphasize that the evaluation is performed from a user's perspective and not from the expert's own perspective.

NOTE: The user was assumed to be an experienced smartphone user and to have knowledge on the use of apps in general. However, this app was used for the first time.

4. Start the screen and audio recording.

5. Have the expert complete the cognitive walkthrough while using the app and carrying out a predetermined set of tasks ${ }^{31}$ : 1) "I want to record my dinner. I started with a cup of tomato soup and a glass of milk.", 2) "Thereafter, I ate a pasta dish, which I consume regularly and want to enter it as a favorite (i.e., predecessor of My Dishes)." [recipe was provided], 3) "As I also consumed the pasta dish, I want to add this to today's food intake record.", and 4) "I entered everything I ate during dinner. I want to check my entry once more and then submit it.".

NOTE: While performing the tasks, the expert informs the researcher about his/her thought 
process, i.e., by explaining the steps needed to be completed to fulfill the described task.

6. Conduct a brief follow-up to clarify ambiguities ${ }^{32}$, and provide the expert the opportunity for additional feedback.

7. Evaluate the results of each expert by checking the recordings to ensure that tasks were executed as intended and by reviewing the additional comments provided

8. Share the results with the experts to assess whether assumptions made based on the recordings were correct.

NOTE: Results of the evaluation were discussed and prioritized in consultation with the experts. Based on the results of this evaluation, the app was further upgraded.

2. Conduct usability testing with intended users to evaluate the app's usability and likability among the intended users by means of think-aloud interviews and the system usability scale (SUS) ${ }^{33}$ by following these steps:

1. Recruit participants who are representative of the target user population ${ }^{35}$.

2. Instruct the participant regarding the study procedures, including the recording of screen and audio. Then, obtain informed consent from the participants.

NOTE: It is important that the researcher encourage the participant to "think-aloud" during the evaluation, i.e., explaining their thoughts on the required steps to complete each task whilst performing the task, as well as commenting on what functionalities did or did not work well.
3. Install the app on the participant's smartphone.

NOTE: To ensure proper installation and functioning and minimize the risk on interruptions during the evaluation, it is recommended that the researcher first verify the app's functionality.

4. Ask the participant to perform a practice task for the think-aloud interview: ask participants to visualize their bedroom and count the number of windows, while telling the researcher about what they saw and thought while counting the windows. Next, ask the participants to approach one of the windows in their bedroom and describe their experiences on their way to that window.

NOTE: A practice task was provided and repeated if needed to ensure that participants felt comfortable to think-aloud as desired ${ }^{37}$.

5. Start the screen and audio recording.

6. Ask the participant to complete actual think-aloud interview with the predefined tasks: the participant must: 1) record everything they ate and drank during the previous day, and 2) record a regularly consumed dish through the My Dishes function.

7. During the session, observe, take notes, and stimulate the participants to keep thinking aloud, if needed, by simple prompts such as "Keep talking out loud", "Tell me what you think", or "Tell me what is on your mind". Minimize further interactions to prevent interference with the participant's thought process $^{28,32}$.

8. Conduct a brief follow-up to clarify ambiguities ${ }^{32}$.

9. Ask the participant to complete an evaluation questionnaire with general questions related to age, 
sex, educational level, type of smartphone, level of smartphone experience (i.e., experienced users are more likely to perform tasks quick and correctly ${ }^{38}$ ), as well as the $S \mathrm{~S}^{33}$-a 10 -item questionnaire to assess the system's usability by means of Likert scale scoring ranging from 1 (strongly disagree) to 5 (strongly agree).

10. Analyze the data from each session by 1) transcribing, coding, and creating (sub)themes, and 2) calculating the SUS score using a predefined formula resulting in a score between 0 to $100^{33}$, where a score of $>68 / 100$ indicates that the tool functions at above-average level of usability and a score $>80 / 100$ indicates excellent usability 39,40 .

NOTE: It is recommended that the researcher who guided the session analyze the data by using qualitative data analysis software. A second researcher can be consulted in case of ambiguities.

3. Conduct quantitative validation of dietary intake records against validated traditional methods and preferably independent measures ${ }^{3}$.

NOTE: The app is being validated against web-based and telephone-based (i.e., interviews) $24 \mathrm{hRs}$ as well as independent urinary and blood biochemical markers. As the quantitative validation of the app is outside the scope of this paper, this will not be discussed further.

\section{Using the backend system for app and study management}

NOTE: The system has three authorization levels: (1) administrator-this authorization level provides access to all sections of the backend (i.e., creating new users, determining user authorization, and granting users access to one or more projects); (2) project managers-this authorization level allows access to specific projects and the possibility to create new projects; and (3) researchers-this authorization level only provides access to the specific projects that researchers are involved in.

1. Management of users and projects in the backend by administrators

1. Access the online backend via traqq.idbit.net, with login credentials (i.e., username, password).

2. Create a new project by clicking on the Projects tab and then on Create a new project.

3. In the next screen, enter the requested project details (i.e., project name, contact description, contact email, contact phone, contact website).

NOTE: Only the project name is mandatory to create a new project. The contact description, email, phone number, and website will become visible in the app under the Contact \& Info button.

4. Select the desired features (i.e., product list, ask eating occasion and/or time of consumption, record or recall).

NOTE: Each new project requires individual decision-making with respect to the most appropriate dietary assessment method (i.e., record or recall), food list, portion size estimation, and eating occasion or meal times.

5. Save the new project by clicking on Save.

NOTE: When the screen closes, the administrator returns to the Project overview screen.

6. Next, create a new user by clicking on the User tab and then on Add new user. 
7. In the following screen, enter a Username, a Password, and assign the user a Role (i.e., administrator, manager, or user).

8. Save the new user by clicking on Save.

NOTE: When the screen closes, the administrator returns to the User overview screen.

9. Assign a user to a project by clicking on the notepad icon (i.e., Edit column) for a specific user.

10. Assign a project by opening the dropdown menu under Linked Projects, selecting the desired project, and clicking on Add.

NOTE: This action needs be repeated for each project the user needs to be assigned to.

11. Communicate the log-in credentials to the new user along with the backend URL.

2. Management of projects in the backend by researchers (i.e., Manager or User role)

1. Log in to the backend via traqq.idbit.net by using the credentials provided by the administrator.

2. Click on Go to projects to manage the projects.

3. Click on the arrow in the View column for the desired project.

NOTE: After doing this, the researcher is taken to a Project Overview page, and new tabs for this specific project appear.

4. Enter the participants in the backend by clicking on the Participants tab. Next, when a Participant Overview screen appears, click on Add new participant.

5. In the following screen, enter Codename, Notes (optional), Login ID, Login Key, and ending with Save.
NOTE: It is recommended that the Participant's study ID be used as both codename and login ID. This minimizes confusion for the participant in case of multiple login credentials. Moreover, the codename is visible in the responses. Using the participant ID makes it easy to use the data. This option needs to be repeated for each participant. For larger groups, Import participants from file (.csv) can be used. Here, the same details are required for each participant. The backend may not contain any personal information of participants.

6. Schedule invitations for each participant by clicking on the Invitations tab. Next, when an Invitation Overview screen appears, click on Add new invitation.

7. In the following screen, select a Participant from the dropdown menu, and enter Period start time, Period end time, Opening time, Closing time, Survey URL (i.e., optional for implementation of additional questions), Notes (optional), Enable (always yes).

NOTE: The Period start and end time refer to reporting time frame (i.e., what has been consumed between ..... and .....). In contrast, opening and closing time refer to the period in which the participant can actually report their intake. The correct implementation of an external survey requires some coding; for this, help from the administrator is recommended. For the majority of the invitations, the Import invitations from (.csv) option under File can be used. The file requires the same information as for the manual input. Invitations can also be created via Sampling Schemes (i.e., where the system generates a random invitation 
scheme across different days and times based on a preset of rules such as sampling period, number of required invitations, response deadline). An advantage of the Sampling Schemes option is that the system automatically schedules a new invitation in case of non-response.

8. Track data collection via the Calendar tab by selecting a participant of interest from the dropdown menu.

NOTE: The calendar provides an overview of scheduled invitations within a project, either in general or for specific participants. Future invitations are portrayed in blue, completed past invitations are green, while past invitations without response are red. Responses to invitations can also be checked via the Response tab.

9. Track responses via the Response tab.

NOTE: In the Response section, the reported food intake data (i.e., food item, consumed amount, eating occasion and/or time of consumption) is gathered.

10. Requests the administrator for data export.

NOTE: Data can be exported from the backend to a .csv file for further analysis (e.g., responses/food intake data, compliance data) by the administrator. Responses include reported food items, selected portion sizes, consumed amounts in grams, and eating occasions/times.

11. Import the .csv file into nutrition calculation software for in-depth nutrient analyses.

NOTE: The data can be imported into nutrition calculation software that makes use of the Dutch FCDB.

\section{Use of the app by the participants during the study}

1. Download the freely available app from the App Store (iOS) or Google Play Store (Android), and access the app by logging in.

NOTE: Login credentials, as provided by the researcher, are required to access the app (step 4.2.5.). After logging in, the app sends invitations as scheduled in the backend based on the participant's credentials (step 4.2.7.).

2. After receiving an invitation via the app, report food intake.

NOTE: Participants can only register their food intake on predetermined days and times.

1. Open the app by clicking on the notification received or by opening the app via the app icon.

NOTE: After opening the app, an Invitation Overview screen appears where previous and current invitations are displayed.

2. Click on the open invitation.

NOTE: Participant is taken to an Overview screen where the invitation period is visible.

3. Enter the food item consumed first by clicking on Product toevoegen (Add food item).

NOTE: The participant is taken to the Search screen.

4. Start typing the name of the consumed item (e.g., orange juice [jus $d$ 'orange]). Click on the desired item as it appears whilst typing.

5. In the following screen, report the consumed amount (Hoeveelheid), corresponding portion size description (Portie), eating occasion 
(Maaltijdmoment) and/or time of consumption (Tijdstip), and end by saving (Opslaan) .

6. Repeat the aforementioned steps until all food items are reported.

7. Submit the list (recall) by clicking on (Lijst versturen) (Send List), or the invitation automatically closes at the end of the day (record). NOTE: The Send List option is also visible in the record version, so participants using the record can also send their input to the database. However, even if the data is already sent, the invite still closes at the end of the day, sending all data to the server.

\section{Representative Results}

The system (app and backend) was developed using the steps outlined in the above described protocol; the key results of this process are described below, concluding with the final design of the app.

\section{Formative research}

In addition to extensive literature review, several webbased tools were inspected (e.g., Compl-eat ${ }^{13}$, ASA24 ${ }^{14}$, Foodbook $24^{15}$, MyFood $24^{16}$ ) with respect to dietary assessment methodology and implemented features. In addition, the performance of several food tracking apps frequently used in the Netherlands were compared (e.g., MijnEetmeter ${ }^{17}$, MyFitnessPal ${ }^{18}$, Virtuagym Food ${ }^{19}$ ), focusing on aspects such as dietary assessment methodology, provision of information, reliability, search engine, and the use of additional features (e.g., images, barcode scanner, recipe functions). The results of this inspection led to the decision to develop the app in such a way that it can be used as a food record and a recall. Moreover, it led to the implementation of the My Dishes function, which can be used to create original recipes or frequently consumed product combinations (e.g., a daily breakfast). Within this function, yield and retention factors are automatically taken into account.

To accurately quantify food and nutrient intake, a complete, albeit practical, food list is crucial. Compiling such a food list requires a trade-off between the extensiveness of the food list and the searchability of the food items (i.e., food descriptions need to be clear, understandable, and easy to locate $)^{41,42}$. As food composition data form the fundamental basis for dietary assessment ${ }^{21,22}$, it is important to ensure that the developed food list can be linked to accurate food composition data. The food list included in the app is based on the Dutch FCDB (NEVO) ${ }^{14}$, which was selected for its reliability and rich food composition data. Originally, the NEVO consists of 2,389 food items (version 2016/5.0), which was reduced to a food list of 1,449 items by eliminating "confusing items" (e.g., foods that cannot be consumed raw, foods that cannot be consumed without additions) or items that are not as essential to include (e.g., due to low consumption rates based on the Dutch Food Consumption Survey (DNFCS) ${ }^{43}$ ).

Additionally, the NEVO contains similar foods with different brand names; in such a case, only the generic option was included in the food list. To further facilitate usability, some food items were renamed to eliminate needless terminology such as 'prepared', 'frozen', 'average', and 'natural'. This "cleaning protocol" was developed by three well-trained research dietitians and executed by means of a syntax, which can be rerun once NEVO is updated. In addition, to optimize the searchability of food items, 1,019 well-known synonyms of the included foods were added to the food list. Thus, the food list included in the app eventually comprised 2,468 items. An overview of the food list development is displayed in Figure 2. To note, although this extensive food list has been developed 
for general use, the backend of the app does allow the import

of alternative food lists if required.

\section{Food Composition Database (FCDB)}

Consists of well-known food items with their

respective food composition data per $100 \mathrm{~g}$

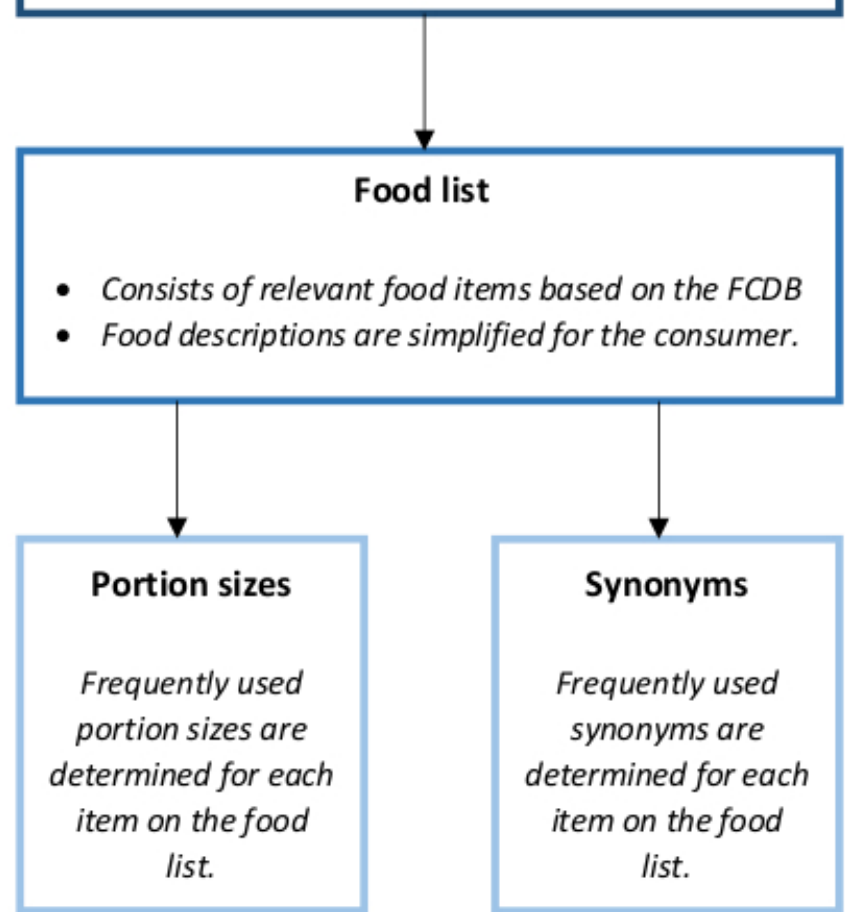

Figure 2: Structure of the food list developed for the app. The food list is based on the Dutch food composition database (FCDB) and corresponding portion size suggestions and synonyms were added for each item in the final food list. Please click here to view a larger version of this figure.

Another crucial aspect of dietary assessment is the quantification of portion sizes. Although portion size estimation aids (PSEAs), e.g., images, referent objects, and standard portion sizes, support the reporting of the amounts of foods consumed $24,26,44$, misreport of portion sizes is still a substantial source of bias $^{24,25,45,46}$, and literature on the effectiveness of the different PSEAs is inconsistent ${ }^{26}$. Food images, portion size suggestions (i.e., standard sizes and household measures), and free entry of weight in grams are the most used PSEAs in web- and smartphone-based dietary assessment tools ${ }^{34}$. For example, whereas portion size suggestions (e.g., cups, spoons, small, large) are used in tools such as Compl-eat ${ }^{13}$ and Oxford $\mathrm{WebQ}^{47}$, images aid portion size estimates in tools such as $\mathrm{ASA} 24^{14}$ and Myfood24 ${ }^{16}$. To investigate the most appropriate PSEA for the app, a pilot study was conducted to compare the accuracy 
of portion size suggestions (e.g., small, medium, large, or cup, spoon), free entry in grams, and portion size images. The results of this study led to the implementation of portion size suggestions as the PSEA in the app along with the option to enter amounts in grams ${ }^{27}$.

\section{Expert review}

The aim of the expert evaluations was to qualitatively evaluate the app in terms of functionality and ease of learning. As many users prefer to learn software by exploration ${ }^{29}$, a system's level of learnability is important. A total of 10 experts, i.e., 4 (research) dietitians and 6 nutrition and health behavior experts (scientists) participated in the cognitive walkthroughs in which $60 \%$ used an Android smartphone. Most importantly, expert evaluations indicated that the first version of the app was not sufficiently intuitive, e.g., menu-structure was judged unclear due to vague buttons/icons, and the search engine generated an illogical order of results. Another critical point arising from the expert reviews related to the fact that selected items could not be modified. Based on these results, the design of the app was considerably upgraded from stage 2 onwards (Figure 1).

\section{Usability evaluation}

A total of 22 participants participated in the think-aloud interviews, which formed the basis of the usability evaluation. The initial sample size was set at 20 participants $^{36}$, after which data saturation was assessed. As data saturation was not reached after 20 interviews, inclusion continued while assessing data saturation after each successive interview. Participants had a mean \pm standard deviation age of $48 \pm 17$ years (range $22-70$ years); $36 \%$ were male, and the majority of the population was highly educated (55\%). In addition, most participants used an Android device ( $n=14,64 \%)$, and almost all participants had over 1 year of experience with smartphone use $(n=21,96 \%)$ (Table 1). All participants completed the tasks without or with minimal instruction.

\begin{tabular}{|c|c|}
\hline & Total $(n=22)$ \\
\hline Gender & 36.4 \\
\hline Male (\%) & 63.6 \\
\hline Female (\%) & $48.1(17.2)$ \\
\hline Mean age (mean, SD) & 0 \\
\hline Educational level & 45.5 \\
\hline Low (\%) & 54.5 \\
\hline Medium (\%) & 63.6 \\
\hline High (\%) & 36.4 \\
\hline Smartphone type & \\
\hline Android (\%) & \\
\hline iOs (\%) & \\
\hline Smartphone experience & \\
\hline
\end{tabular}




\begin{tabular}{|c|c|}
\hline Shorter than 6 months (\%) & 4.5 \\
\hline Between 6 months and 1 year (\%) & 0 \\
\hline Longer than 1 year (\%) & 95.5 \\
\hline SUS (mean, SD) & $79.4(15.1)$ \\
\hline
\end{tabular}

Table 1. Characteristics of the study population and results of usability evaluation. Only the results of the system usability scale (SUS) are portrayed in this table along with the participant characteristics.

Whereas some participants $(n=13,59 \%)$ indicated difficulties while using the My Dishes functionality; others $(n=5$, $23 \%$ ) encountered minor functionality issues such as slow response of the menu button and difficulties using buttons related to insufficient screen size of smaller smartphones). Moreover, 15 (68\%) participants indicated their preference for an option to enter consumed portion sizes in grams. Finally, evaluation of the SUS score indicated a rating of 79/100 (range 40-100), wherein only 3 out of the 22 participants rated the app below $68 / 100$ and 13 rated $>80 / 100$, which suggests that the app can be considered user-friendly. Thus, overall, the suggested improvements were minor, and usability evaluations were promising. Subsequently, suggestions for improvement were discussed within the research team and, if deemed relevant, incorporated in the stage 4 upgrade to further optimize likability and usability of the app (Figure 1).

\section{Final design}

The steps described in the protocol and the results of the evaluation study eventually resulted in a final design for the app and the backend, which aimed for a simple visual design. This app can be used as a food record and a recall. As described previously, the food list is a modified version of the NEVO. Portion size estimation is supported by foodspecific portion size suggestions; consumed portions can also be entered in grams. In case of the recall version of the app, the researcher has the possibility to select different timeslots (e.g., $2 \mathrm{hR}, 8 \mathrm{hR}$, or $24 \mathrm{hR}$ ). To collect food intake data on different days and times, various sampling schemes can be created within a predetermined period. Push notifications invite respondents to record their food intake. To ensure complete data collection, invitations are automatically rescheduled in case of non-response. Within the recall module, respondents can only report their food intake after receiving an invitation. In case of the food record, respondents can access the app and log their food intake throughout the day.

In contrast to most $24 \mathrm{hR}$ tools, the recall module of the app is not based on the Automated Multiple-Pass Methoda five-step method for collecting food intake data for the previous $24 \mathrm{~h}^{48}$-as this method is too elaborate and timeconsuming for use in an app. More specifically, to increase usability and enhance the compliance of the food intake recordings $11,38,49$, navigation was reduced to a minimum by limiting the number of screens that need to be accessed to 4 (Figure 3): 1) an Overview screen showing the reporting window; 2) consumed food items are reported through the Search screen, and once the desired item is selected 3) a dialog box appears probing eating occasion and consumed amount, after which 4) the user returns to the Overview screen now showing the recorded food items. In addition, the 

button.

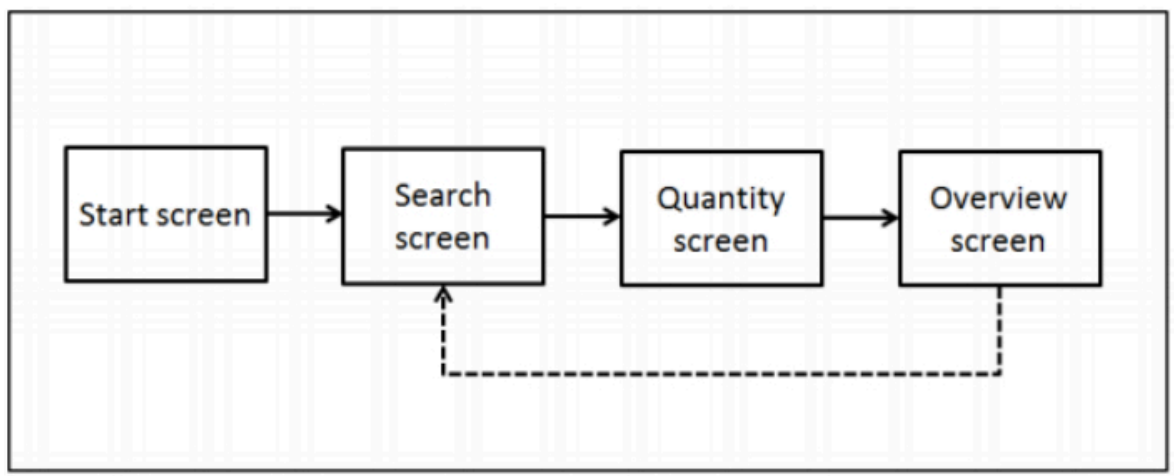

Figure 3: Schematic overview of the routing in the app. Please click here to view a larger version of this figure.

The data are stored on a secure server. If desired, additional questions-general or related to specific eating occasions or food items-can be incorporated. The app can connect with online survey tools. Therefore, it is possible to conduct a survey unrelated to food intake via the app at prespecified times (e.g., context, behavioral, mood questions). It is also possible to ask specific questions related to reported food items or eating occasions (e.g., when apples are reported, when lunch is reported). The use of online survey tools provides an opportunity to ask many different questions via the app. The collected food intake data can be exported from the server and imported into nutrition calculation software for further analyses. In case of the use of additional questions, these data will be available in the survey tool as usual. The aim was to develop a well-structured and easy-to-use app. Some screenshots of the design can be seen in Figure 4A-E. 


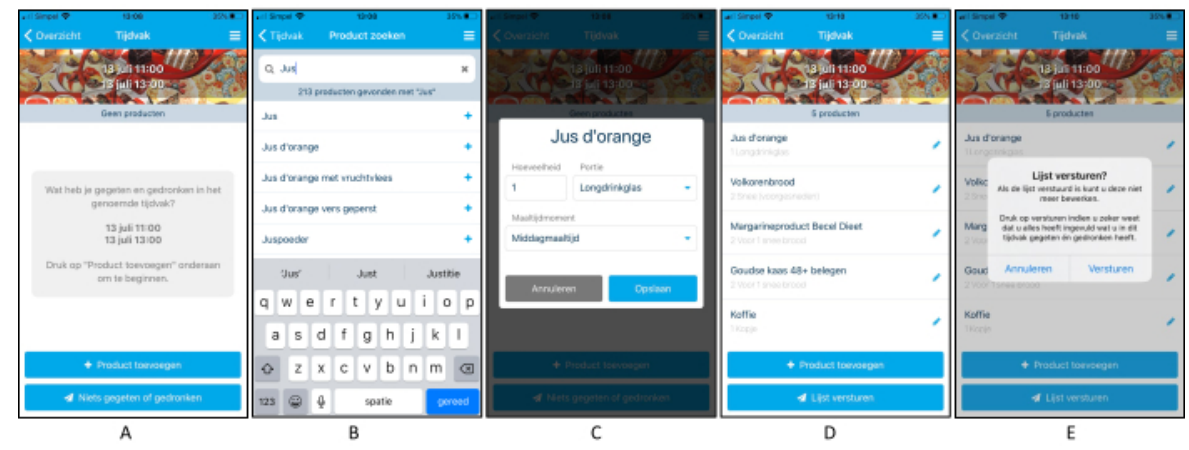

Figure 4: Screenshots of the final version of the app. (A) The Start/Overview screen, showing the invitation with the (in this case) 2 h-recall period. The user can press Product toevoegen (i.e., Add item) to report a food item or Niets gegeten of gedronken (i.e., I did not eat or drink anything) in case nothing was consumed during this time window. (B) The Search screen, showing results matching the search term "Jus" from the food list. The desired item can be selected from the search results. (C) A pop-up screen requires input of details on the selected item "Jus d 'orange". In this case, the app asks for the amount consumed and eating occasion. The user can go back to the search result by pressing Annuleren (i.e., cancel) or Opslaan (i.e., save) to go further. (D) The Overview again, this time showing all the reported items. Another item can be added (Product toevoegen) or the input can be sent (Lijst versturen). (E) After selecting Lijst versturen, a pop-up appears asking the user if they are sure that they want to send, and reminds the user that it is not possible to make any more changes after the list has been sent. The user has the option to cancel (Annuleren) or send (Versturen). Please click here to view a larger version of this figure.

\section{Discussion}

This paper presents the iterative developmental process of the smartphone-based dietary assessment app Traqq. Balancing the required level of accuracy and user-friendliness posed the following main challenges in the development of the app related to decisions on 1) data entry (i.e., selecting the most accurate method for food identification and portion size quantification), 2) food composition data (i.e., selecting an accurate database and creating a fullfledged food list), 3) customization options (i.e., flexibility in food list, portion size quantification, and recipes), and 4) validation (i.e., against traditional methods and/or independent measures) $)^{3,50}$. During the literature review, five validated and fully automated, smartphone-based, dietary assessment tools developed for research were identified $^{3}$, namely My Meal Mate ${ }^{4}$, Electronic Dietary Intake Assessment $(\mathrm{eDIA})^{7}$, Easy Diet Diary ${ }^{8}$, Electronic Carnet Alimentaire (e-CA $)^{5}$, and Eat and Track (EaT) ${ }^{6}$.

Owing to the level of automatization of these five dietary assessment apps as well as this app, researcher burden and costs substantially decreases while data completeness increases compared to traditional dietary assessment methods. Additionally, this app, in turn, differs from the five existing dietary assessment tools in terms of flexibility. 
Specifically, whereas existing apps are all based on the food record method, this app can be used as a food record as well as a recall. Moreover, whereas the design of these apps is fixed, Traqq has the major advantage that it can be modified to fit different research purposes (e.g., dietary assessment method, food list, sampling schemes, additional questions) $)^{3,50}$. Conversely, other existing dietary assessment apps contain valuable features, which are not implemented in the app (yet). To illustrate this point, some apps allow the user to take photographs of their food for food recognition and portion size estimation such as the semiautomated, technology-assisted dietary assessment (TADA) system $^{51,52}$.

Participants in the usability study also indicated that the use of photographs could be a valuable addition to aid portion size estimation. However, there were still too many challenges to be addressed to implement such a feature at this stage, e.g., specifying and guiding with respect to the photographic angle (i.e., to assess depth), the need for a reference maker (i.e., to correct for sizes and colors), the essential before and after photo (i.e., to assess consumed amounts), and on how to process recipe dishes. Due to these technical challenges, the existing image-based dietary assessment apps are still semi-automated, which means manual image review must be done by the user, the researcher, or both ${ }^{51,52}$. Technological advances, such as crowdsourcing and machine learning, have the potential to improve the use of food images for dietary assessment ${ }^{53,54}$. In the future, these options will be explored to further improve the app. The developmental process of the app was characterized by various critical steps. First, a formative research step was completed wherein the scientific concepts underpinning the rationale for app creation facilitated decision-making in setting up the general outline of the app.

During this stage, special attention was paid to the selection of the FCDB and the selection of the PSEA-aspects that both directly influence data accuracy ${ }^{21}$. Regarding the FCDB, as the app has originally been developed for use in the Netherlands, its food list is based on the Dutch FCDB, $\mathrm{NEVO}^{14}$. In the future, the aim is to further develop the app for international use, which requires more extensive food composition data as many foods are country-specific. Currently, no international FCDB exists yet and if existent, its use might have been limited. More specifically, as the Dutch food list already contains 2,389 food items, the implementation of an international food composition table, e.g., for 5 countries would probably multiply this number of food items by about 5 and negatively affect the searchability of foods and consequently, the app's usability. Therefore, country-specific food lists will probably be most valuable and often also preferred by professionals ${ }^{55}$.

This is facilitated by the app as it enables the import of alternative food lists and thus linkage to different (international) food composition tables. Regarding the portion sizes, there are multiple options available to support the accuracy of the estimates, e.g., use of image booklets, referent objects, and/or textual portion size suggestions ${ }^{26}$. In view of user-friendliness, direct implementation of a PSEA in the app is preferred over using a PSEA alongside the app (e.g., image booklet, referent objects). During the development of the app, the decision was made to facilitate portion size quantification by offering the opportunity to enter portion sizes using portion size suggestions and entry in grams. Portion size suggestion are based on the only available Dutch portion size database ${ }^{56}$. Although Dutch 
dietary assessment tools such as Compl-eat and Eetmeter also rely on this database $\mathrm{i}^{13,17}$, it needs to be noted that this portion size database dates from 2003 , and tableware sizes have since increased ${ }^{57}$. Using this database may therefore underestimate food intake.

Currently, the portion size database is being updated by the Dutch National Institute for Public Health and the Environment (RIVM), the Dutch Nutrition Center, and Wageningen University and Research ${ }^{58}$, which will eventually be used to update the portion size suggestions in the app. Discrepancies between the old and new portions will be mapped and adjusted where needed. Although the use of portion size images (i.e., a series of images portraying different amounts of a selected food) may be a good alternative for textbased portion size suggestions ${ }^{59}$, research has shown that the accuracy of portion size estimation is highest when a series of portion size images is presented at once, instead of one image at a time $45,60,61$. Generally, currently available smartphones have relatively small screens, which limits the presentation of a series of images. Although new technologies facilitate the use of interactive portion size graphics wherein amounts of food on a virtual plate or cup can be increased or decreased by using a slider ${ }^{62}$, these techniques are relatively new and still need to be thoroughly evaluated to assess their accuracy.

Another critical step in the development of the app included the involvement of experts and intended end-users. Although not often incorporated in the developmental process of tools (or not described) ${ }^{11,12}$, feedback from experts-as well as intended end-users-is crucial ${ }^{63}$, allows maximization of usability, and maintains the required level of accuracy. The feedback of the intended end-users was particularly helpful in the final design of the My Dishes function. Overall, the users were satisfied with the possibility to create their own dishes. However, they did struggle with some of the procedures, for instance, although the function would automatically save data, this was not visible to the user. Therefore, many users kept searching for the Save button and got stuck, afraid to go back and lose their input. Based on these kinds of feedback, the function was improved to better fit the expectations of the user.

To conclude, Traqq is an innovative app with many advantages over existing apps and web-based tools. However, there are still various limitations. As the app still relies on self-report, self-report-related measurement errors still exist (e.g., memory bias (i.e., in case of recall), social desirability bias, and food intake modifications (i.e., in case of food records), inaccurate portion size estimations (i.e., in both) $)^{1}$. In the coming years, recently launched novel technologies will be explored to further advance the app, e.g., by exploring the value of implementing features such as barcode scanners, voice recording, chatbots, and images, which could improve food identification and portion size estimation. Possibilities to connect with other apps (e.g., activity trackers, sleep trackers) and devices (e.g., accelerometers, heart rate monitors, chewing sensors) are being explored as well. Finally, the backend is also being subjected to further development e.g., through the expansion of sampling options.

\section{Disclosures}

The authors have nothing to disclose.

\section{Acknowledgments}

The authors want to thank Anouk Geelen and Arvind Datadien for their key role in the development of Traqq. 
Furthermore, the authors would like to thank Romy

Willemsen for her assistance in the data collection and the data analysis in the usability study. Finally, the authors want to thank the experts and participants for sharing their experiences and opinions throughout the process. The development was executed by Wageningen University and Research and partly funded by the Ministry of Agriculture, Nature and Food Quality and industry, in the context of TKI Agri\&Food PPS - project Smart Food Intake (AF16096).

\section{References}

1. Brouwer-Brolsma, E. M. et al. Dietary intake assessment: From traditional paper-pencil questionnaires to technology-based tools. In Environmental software systems. Data science in action. ISESS 2020. IFIP Advances in Information and Communication Technology. Athanasiadis, I., Frysinger, S., Schimak, G., Knibbe, W. (Eds), 554, Springer, Cham, Chapter 2, 7-23 (2020).

2. Thompson, F. E., Subar, A. F. in Nutrition in the prevention and treatment of disease. Coulston, A., Boushey, C., Ferruzzi, M., Delahanty, L. (Eds), Elsevier, Inc., 5-48 (2017).

3. Eldridge, A. L. et al. Evaluation of new technologybased tools for dietary intake assessment-an ILSI Europe Dietary Intake and Exposure Task Force Evaluation. Nutrients. 11 (1), 55 (2018).

4. Carter, M. C., Burley, V. J., Nykjaer, C., Cade, J. E. 'My Meal Mate' (MMM): validation of the diet measures captured on a smartphone application to facilitate weight loss. British Journal of Nutrition. 109 (3), 539-546 (2013).

5. Bucher Della Torre, S., Carrard, I., Farina, E., Danuser, B., Kruseman, M. Development and Evaluation of e-CA, an electronic mobile-based food record. Nutrients. 9 (1), 76 (2017).

6. Wellard-Cole, L. et al. Relative validity of the Eat and Track (EaT) smartphone app for collection of dietary intake data in 18-to-30-year olds. Nutrients. 11 (3), 621 (2019).

7. Rangan, A. M. et al. Electronic Dietary Intake Assessment (e-DIA): Comparison of a mobile phone digital entry app for dietary data collection with 24-hour dietary recalls. JMIR mHealth and uHealth. 3 (4), e98 (2015).

8. Ambrosini, G. L., Hurworth, M., Giglia, R., Trapp, G., Strauss, P. Feasibility of a commercial smartphone application for dietary assessment in epidemiological research and comparison with 24-h dietary recalls. Nutrition Journal. 17 (1), 5 (2018).

9. Subar, A. F. et al. Addressing current criticism regarding the value of self-report dietary data. The Journal of Nutrition. 145 (12), 2639-2645 (2015).

10. Kipnis, V. et al. Bias in dietary-report instruments and its implications for nutritional epidemiology. Public Health Nutrition. 5 (6A), 915-923 (2002).

11. Simpson, E. et al. Iterative development of an online dietary recall tool: INTAKE24. Nutrients. 9 (2), 118 (2017).

12. Chen, J., Cade, J. E., Allman-Farinelli, M. The most popular smartphone apps for weight loss: a quality assessment. JMIR Mhealth Uhealth. 3 (4), e104 (2015).

13. Apple Inc. Human Interface Guidelines., https:// developer.apple.com/ios/human-interface-guidelines/ (2017).

14. RIVM. NEVO-online (2016/5.0). (2016). 
15. Jaspers, M. W. A comparison of usability methods for testing interactive health technologies: methodological aspects and empirical evidence. International Journal of Medical Informatics. 78 (5), 340-353 (2009).

16. Penha, A. D. S., dos Santos, F. A. N. V. Evaluating the use of the cognitive walk-through usability. Human Factors in Design. 1 (1) (2012).

17. Fonteyn, M. E., Kuipers, B., Grobe, S. J. A description of think aloud method and protocol analysis. Qualitative Health Research. 3 (4), 430-441 (2016).

18. Brooke, J. SUS - A quick and dirty usability scale. Usability evaluation in industry. 189 (194), 4-7 (1996).

19. Kushniruk, A. W., Patel, V. L. Cognitive and usability engineering methods for the evaluation of clinical information systems. Journal of Biomedical Informatics. 37 (1), 56-76 (2004).

20. Davison, G. C., Vogel, R. S., Coffman, S. G. Think-aloud approaches to cognitive assessment and the articulated thoughts in simulated situations paradigm. Journal of Consulting and Clinical Psychology. 65 (6), 950-958 (1997).

21. Moumane, K., Idri, A., Abran, A. Usability evaluation of mobile applications using ISO 9241 and ISO 25062 standards. SpringerPlus. 5, 548 (2016).

22. Sauro, J. A practical guide to the system usability scale: Background, benchmarks \& best practices. CreateSpace Independent Publishing Platform (2011).

23. Bangor, A., Kortum, P., Miller, J. Determining what individual SUS scores mean: Adding an adjective rating scale. Journal of Usability Studies. 4 (3), 114-123 (2009).

24. Meijboom, S. et al. Evaluation of dietary intake assessed by the Dutch self-administered web-based dietary 24-h recall tool (Compl-eat ${ }^{\mathrm{TM}}$ ) against intervieweradministered telephone-based 24-h recalls. Journal of Nutritional Science. 6, e49 (2017).

25. Subar, A. F. et al. The Automated Self-Administered 24hour dietary recall (ASA24): a resource for researchers, clinicians, and educators from the National Cancer Institute. Journal of the Academy of Nutrition and Dietetics. 112 (8), 1134-1137 (2012).

26. Timon, C. M. et al. The development, validation, and user evaluation of Foodbook24: A web-based dietary assessment tool developed for the Irish adult population. Journal of Medical Internet Research. 19 (5), e158 (2017).

27. Carter, M. C. et al. Development of a UK online 24h dietary assessment tool: myfood24. Nutrients. 7 (6), 4016-4032 (2015).

28. Voedingscentrum. Mijn Eetmeter., https:// itunes.apple.com/nl/app/mijn-eetmeter/id663382012? $\mathrm{mt}=8$ (2014).

29. MyFitnessPal. MyFitnessPal., http:// www.myfitnesspal.com/welcome/learn_more (2015).

30. Virtuagym Food. Virtuagym Food., https:// virtuagym.com/food (2017).

31. Evans, K. et al. Development and evaluation of a concise food list for use in a web-based 24-h dietary recall tool. Journal of Nutritional Science. 6, e46 (2017).

32. Svensson, A., Magnusson, M., Larsson, C. Overcoming barriers: adolescents' experiences using a mobile phone dietary assessment app. JMIR mHealth and uHealth. 4 (3), e92 (2016).

33. Finglas, P. M., Berry, R., Astley, S. Assessing and improving the quality of food composition databases 
for nutrition and health applications in Europe: the contribution of EuroFIR. Advances in Nutrition. 5 (5), 608S-614S (2014).

34. Gibson-Moore, H. EuroFIR: Where we are now? Nutrition Bulletin. 38 (3), 358-362 (2013).

35. Rossum van, C. T. M. et al. The diet of the Dutch. Results of the first two years of the Dutch National Food Consumption Survey 2012.-2016, RIVM (2016).

36. Byrd-Bredbenner, C., Schwartz, J. The effect of practical portion size measurement aids on the accuracy of portion size estimates made by young adults. Journal of Human Nutrition and Dietetics. 17 (4), 351-357 (2004).

37. Faggiano, F. et al. Validation of a method for the estimation of food portion size. Epidemiology. 3 (4), 379-382 (1992).

38. Faulkner, G. P. et al. An evaluation of portion size estimation aids: precision, ease of use and likelihood of future use. Public Health Nutrition. 19 (13), 2377-2387 (2016).

39. Hernandez, T. et al. Portion size estimation and expectation of accuracy. Journal of Food Composition and Analysis. 19, S14-S21 (2006).

40. Nelson, M., Atkinson, M., Darbyshire, S. Food photography. I: The perception of food portion size from photographs. British Journal of Nutrition. 72 (5), 649-663 (1994).

41. Young, L. R., Nestle, M. S. Portion sizes in dietary assessment: issues and policy implications. Nutrition Reviews. 53 (6), 149-158 (1995).

42. Liu, B. et al. Development and evaluation of the Oxford WebQ, a low-cost, web-based method for assessment of previous $24 \mathrm{~h}$ dietary intakes in large-scale prospective studies. Public Health Nutrition. 14 (11), 1998-2005 (2011).

43. Lucassen, D. A., Willemsen, R. F., Geelen, A., BrouwerBrolsma, E. M., Feskens, E. J. M. The accuracy of portion size estimation using food images and textual descriptions of portion sizes: an evaluation study. Journal of Human Nutrition and Dietetics. In press (2021).

44. Wharton, C., Rieman, J., Lewis, C., Polson, P. in Usability Inspection Methods. Nielsen, J., Mack, R. L. (Eds), John Wiley \& Sons, 79-104 (1994).

45. Nielsen, J. How many test users in a usability test?., https://www.nngroup.com/articles/howmany-test-users/ (2012).

46. USDA. AMPM - Features. https://www.ars.usda.gov/ northeast-area/beltsville-md/beltsville-human-nutritionresearch-center/food-surveys-research-group/docs/ ampm-features/ (2016).

47. Zhang, D. S., Adipat, B. Challenges, methodologies, and issues in the usability testing of mobile applications. International Journal of Human-Computer Interaction. 18 (3), 293-308 (2005).

48. Cade, J. E. Measuring diet in the 21st century: use of new technologies. Proceedings of the Nutrition Society. 76 (3), 276-282 (2017).

49. Ahmad, Z. et al. A mobile food record for integrated dietary assessment. MADiMa16 (2016). 2016, 53-62 (2016).

50. Boushey, C. J., Spoden, M., Zhu, F. M., Delp, E. J., Kerr, D. A. New mobile methods for dietary assessment: review of image-assisted and image-based dietary assessment methods. Proceedings of the Nutrition Society. 76 (3), 283-294 (2017). 
51. Fang, S. et al. in 2018 IEEE Southwest Symposium on Image Analysis and Interpretation (SSIAI). Las Vegas, Nevada, USA, 25-28 (2018).

52. Shao, Z., Mao, R., Zhu, F. in 2019 IEEE International Conference on Big Data (Big Data). Los Angeles, California, USA, 5186-5189 (2019).

53. Chen, J., Lieffers, J., Bauman, A., Hanning, R., AllmanFarinelli, M. Designing health apps to support dietetic professional practice and their patients: qualitative results from an international survey. JMIR Mhealth Uhealth. 5 (3), e40 (2017).

54. Donders-Engelen, M. R., Van der Heijden, L. J. M., Hulshof, K. F. A. M. Maten, Gewichten en Codenummers 2003. Food portion sizes and coding instructions. Wageningen University: Division of Human Nutrition and TNO Nutrition (2003).

55. Van Ittersum, K., Wansink, B. Plate size and color suggestibility: The Delboeuf Illusion's bias on serving and eating behavior. Journal of Consumer Research. 39 (2), 215-228 (2012).

56. RIVM. Portiegrootte voedingsmiddelen. https:// www.rivm.nl/portiegrootte-voedingsmiddelen (2019).

57. Timon, C. M. et al. A review of the design and validation of web- and computer-based 24-h dietary recall tools. Nutrition Research Reviews. 29 (2), 268-280 (2016).

58. Kirkpatrick, S. I. et al. The use of digital images in 24hour recalls may lead to less misestimation of portion size compared with traditional interviewer-administered recalls. The Journal of Nutrition. 146 (12), 2567-2573 (2016).

59. Subar, A. F. et al. Assessment of the accuracy of portion size reports using computer-based food photographs aids in the development of an automated self-administered 24-hour recall. Journal of the American Dietetic Association. 110 (1), 55-64 (2010).

60. Figwee. Figwee - Learn More. https://figwee.com/learnmore/ (2021).

61. Preece, J., Sharp, H., Rogers, Y. Interaction design: beyond human-computer interaction. John Wiley \& Sons (2015). 